\title{
Comparative activity of several antimicrobial agents against nosocomial Gram-negative rods isolated across Canada
}

\author{
Shelley R ScRiver Rt, CANadian Antimicrobial Resistance Study Group, Donald E LoW mD fRcpc
}

\begin{abstract}
SR Scriver, Canadian Antimicrobial Resistance Study Group, DE Low. Comparative activity of several antimicrobial agents against nosocomial Gram-negative rods isolated across Canada. Can J Infect Dis 1995;6(2):76-82. In 1992, a surveillance study was performed in Canada to determine the susceptibility of nosocomial Gram-negative rods to several wide spectrum antimicrobials. Consecutive isolates from 10 institutions, as well as additional strains of selected species of Enterobacteriaceae that are known to possess the Bush group 1 beta-lactamase, were tested for susceptibility to 12 antimicrobials. Third-generation cephalosporin resistance was found to be as high as $29 \%$ in Enterobacter cloacae that possesses the Bush group 1 beta-lactamase and less than $4 \%$ in those isolates not possessing this enzyme. Cefepime equalled or exceeded the activity of the third-generation cephalosporins against the species of Enterobacteriaceae that demonstrated resistance to the third-generation cephalosporins.
\end{abstract}

Key Words: Antimicrobial resistance, Gram-negative rods

\section{Activité comparative de plusieurs antibiotiques contre les souches nosocomiales gram-négatives isolées au Canada}

RÉSUMÉ : En 1992, une étude épidémiologique a été effectuée au Canada afin de déterminer le degré de sensibilité des souches nosocomiales Gram-négatives à divers antibiotiques à large spectre. Des isolats consécutifs provenant de dix établissements, de même que d'autres souches d'espèces sélectionnées d'Enterobacteriaceae dont on sait qu'elles sont dotées de bêta-lactamases Bush de groupe 1 ont été soumis à des épreuves de sensibilité à 12 antimicrobiens. Une résistance de l'ordre de $29 \%$ aux céphalosporines de troisième génération a été observée dans une souche d'Enterobacter cloacae dotée de bêta-lactamases Bush du groupe 1, et inférieure à $4 \%$ dans les isolats dépourvus de cet enzyme. La céfépime a manifesté une activité égale ou supérieure aux céphalosporines de troisième génération contre les espèces d'Enterobacteriaceae qui manifestaient une résistance aux céphalosporines de troisième génération.

Department of Microbiology, Mount Sinai and Princess Margaret Hospitals, University of Toronto, Toronto, Ontario

The Canadian Antimicrobial Resistance Study Group comprises: Anne-Marie Bourgault MD FRCPC, Hopital St-Luc, Montreal, Quebec; James Brunton MD FRCPC, The Toronto Hospital, Toronto, Ontario; Kevin Forward MD FRCPC, Victoria General Hospital, Halifax, Nova Scotia; Daryl Hoban PHD, Health Sciences Centre, Winnipeg, Manitoba; Jean R Joly MD FRCPC, St Sacrement Hospital, Ste-Foy, Quebec; Tom J Louie MD FRCPC, Calgary General Hospital, Calgary, Alberta; Vivian Loo MD FRCPC, Royal Victoria Hospital, Montreal, Quebec; Anne Phillips MD FRCPC, The Toronto Hospital, Toronto, Ontario; Robert Rennie PHD, University of Alberta Hospitals, Edmonton, Alberta; and John A Smith MD FRCPC, Vancouver General Hospital, Vancouver, British Columbia

Correspondence and reprints: Dr DE Low, Department of Microbiology, Mount Sinai Hospital, 600 University Avenue, Room 1487, Toronto, Ontario M5G 1X5. Telephone (416) 586 4435, Fax (416) 586 8746, e-mail low@mshri.on.ca

Received for publication August 4, 1994. Accepted October 13, 1994 
$\mathrm{T}$ HE INTRODUCTION OF THIRD-GENERATION CEPHALOsporins has improved our ability to treat serious infections caused by Gram-negative pathogens. However, increased use of these agents has been followed by the emergence of resistance to them. This resistance can be mediated by diminished outer membrane permeability, production of the chromosomally mediated inducible beta-lactamase, acquisition of plasmid mediated betalactamases or combinations of these mechanisms (1-3). Initially, extended spectrum cephalosporins were believed to be stable to both chromosomal and plasmid mediated enzymes. However, since 1985, outbreaks and sporadic cases of infections due to ceftazidime-resistant organisms with plasmidmediated extended spectrum beta-lactamases have been described in Europe, the United States and elsewhere (4-8). Sporadic mutations leading to stable derepression of the chromosomally mediated inducible beta-lactamase also result in resistance to third-generation cephalosporins (1,9-12). These Bush group 1 (BGI) enzymes are generally identified in species of Enterobacter, Citrobacter, Serratia, Providencia, Morganella, indole-positive Proteus and Pseudomonas aeruginosa.

Cefepime is an extended spectrum cephalosporin that is considered to be a 'fourth-generation' cephalosporin. This group of cephalosporins has a slightly different penicillinbinding protein target-binding profile, lower affinity for chromosomally encoded beta-lactamases, particularly BGI, and a higher rate of penetration through outer membrane porins of Gram-negative bacteria compared with other cephalosporins. These differences enhance the spectrum of bactericidal activity of cefepime relative to all available cephalosporins (13). Other studies have demonstrated that Gram-negative isolates that are resistant to one or more third-generation cephalosporins are susceptible to cefepime $(14,15)$. In a recent Canadian survey, it was determined that $23 \%$ of isolates of Enterobacter cloacae were ceftazidime resistant (16).

Tazobactam is a recently studied penicillanic acid sulfone that inhibits a wide variety of lactamases (17-21). The combination of tazobactam with penicillins and broad spectrum cephalosporins has demonstrated broad antimicrobial activity $(19,20)$.

The purpose of this study was to determine the in vitro activity of cefepime and piperacillin/tazobactam, two new antimicrobial agents, in addition to several other antimicrobials against a selected group of nosocomial Gram-negative isolates from several centres across Canada.

\section{MATERIALS AND METHODS}

Bacterial isolates: Approximately 100 consecutive nonduplicate nosocomial isolates of common Gram-negative bacterial pathogens were collected from 10 centres across Canada. Participating centres were asked to submit an additional 50 isolates of species known to harbour BGI beta-lactamases, specifically: E cloacae, Enterobacter species, Citrobacter freundii, Morganella morganii, Proteus vulgaris, Providencia species, Serratia marcescens and $P$ aeruginosa. Isolates were identified using standard methodologies (22) and frozen at $-70^{\circ} \mathrm{C}$ in phosphate buffered glycerol. All isolates were subcultured twice before susceptibility testing.

Susceptibility testing: Broth microdilution was performed according to National Committee for Clinical Laboratory Standards guidelines (23). Microdilution panels were prepared by dispensation of cation-supplemented Mueller-Hinton broth containing twofold-concentration increments of antimicrobial agents in $100 \mu \mathrm{L}$ aliquots into plastic 96-well trays. Inoculum suspensions equal to a $0.5 \mathrm{McF}$ arland standard were further diluted and added to the microdilution trays to achieve a final inoculum of $5 \times 10^{5}$ colony forming units (CFU)/mL. Colony counts were performed to confirm the final inoculum. Following inoculation, microdilution trays were incubated at $35^{\circ} \mathrm{C}$ in ambient air for 16 to $20 \mathrm{~h}$. After incubation, the minimum inhibitory concentration (MIC) was defined as the lowest concentration of antimicrobial agent with no visible evidence of growth. Cefepime (Bristol-Myers Squibb, Connecticut), piperacillin (Lederle Laboratories, New Jersey), tazobactam (Lederle Laboratories), cefotaxime (Hoechst-Roussel Pharmaceuticals Inc, New Jersey), ceftazidime (Glaxo, North Carolina), ceftriaxone (Hoffman-La Roche, Inc, New Jersey), ticarcillin (Beecham Laboratories, Tennessee), clavulanate (Beecham Laboratories) and imipenem (Merck Sharp and Dohme, New Jersey) were obtained from their respective manufacturers. Gentamicin, tobramycin, ciprofloxacin and trimethoprimsulfamethoxazole powders were obtained from Sigma (Sigma Chemical Co, Missouri). Tazobactam was combined with piperacillin at a fixed concentration of $4.0 \mathrm{~g} / \mathrm{mL}$. Clavulanic acid was combined with ticarcillin in a ratio of ticarcillin to clavulanate of $2: 1$.

Escherichia coli ATCC 25922 and $P$ aeruginosa ATCC 27853 were used as control strains for broth microdilution testing.

\section{RESULTS}

From 10 centres across Canada, 1984 isolates of Enterobacteriaceae and $P$ aeruginosa were collected. The distribution of bacterial species collected was E cloacae $(27.7 \%)$, E coli $(18.8 \%)$, Klebsiella pneumoniae (12.4\%), $P$ aeruginosa (11.5\%), S marcescens (9.5\%), C freundii (8.0\%), Enterobacter species (6.7\%), M morganii (2.9\%), $P$ vulgaris (1.2\%), $P$ mirabilis (0.8\%) and Providencia species (05\%). Susceptibility results based on broth microdilution testing are presented in Table 1 as $\mathrm{MIC}_{50}$, MIC90, range and percentage resistance.

Third-generation cephalosporin resistance was rare in species where resistance would be characterized by the presence of plasmid-mediated extended spectrum betalactamases, specifically $E$ coli and $K$ pneumoniae. Rates of resistance to ceftazidime in these isolates were $1.1 \%$ and $2.9 \%$, respectively. However, in isolates with $\mathrm{BGI}$ betalactamases, rates of resistance to the third-generation cephalosporins were significant. E cloacae demonstrated the highest levels of resistance to ceftazidime (28.9\%). Isolates resistant to ceftazidime also demonstrated cross-resistance to other third-generation cephalosporins. The most active cephalosporin tested against all isolates was cefepime. 
TABLE 1

Comparative activity of 12 antimicrobials against Gram-negative clinical isolates from 10 medical centres

\begin{tabular}{|c|c|c|c|c|c|}
\hline \multirow{2}{*}{$\begin{array}{l}\text { Organism } \\
\text { (number) }\end{array}$} & \multirow[b]{2}{*}{ Antimicrobial agent } & \multicolumn{3}{|c|}{$\operatorname{MIC}(\mu \mathrm{g} / \mathrm{mL})^{*}$} & \multirow[b]{2}{*}{ Resistant (\%) } \\
\hline & & $50 \%$ & $90 \%$ & Range & \\
\hline \multirow{12}{*}{$\begin{array}{l}\text { Escherichia coli } \\
\text { (373) }\end{array}$} & Cefotaxime & $\leq 0.06$ & 0.12 & $\leq 0.06-1.0$ & 0 \\
\hline & Ceftazidime & $\leq 0.50$ & $\leq 0.50$ & $\leq 0.50-1.0$ & 1.1 \\
\hline & Ceftriaxone & $\leq 0.50$ & $\leq 0.50$ & $\leq 0.50-1.0$ & 0 \\
\hline & Cefepime & $\leq 0.06$ & $\leq 0.06$ & $\leq 0.06-0.25$ & 0 \\
\hline & Piperacillin & 2.0 & $>512.0$ & $\leq 1.0->512.0$ & 19.0 \\
\hline & Piperacillin/tazobactam & 2.0 & 16.0 & $\leq 1.0->256.0$ & 2.3 \\
\hline & Ticarcillin/clavulanate & 2.0 & 64.0 & $\leq 1.0->256.0$ & 5.8 \\
\hline & Imipenem & 0.25 & 0.50 & $\leq 0.06-4.0$ & 0 \\
\hline & Gentamicin & $\leq 4.0$ & $\leq 4.0$ & $\leq 4.0->16.0$ & 2.9 \\
\hline & Tobramycin & $\leq 4.0$ & $\leq 4.0$ & $\leq 4.0-8.0$ & 1.8 \\
\hline & Ciprofloxacin & $\leq 0.06$ & $\leq 0.06$ & $\leq 0.06-8.0$ & 1.1 \\
\hline & TMP/SMX & $\leq 0.50$ & $>16.0$ & $\leq 0.50->16.0$ & 12.2 \\
\hline \multirow{12}{*}{$\begin{array}{l}\text { Klebsiella } \\
\text { pneumoniae } \\
(247)\end{array}$} & Cefotaxime & $\leq 0.06$ & 0.12 & $\leq 0.06->64.0$ & 1.1 \\
\hline & Ceftazidime & $\leq 0.50$ & $\leq 0.50$ & $\leq 0.50->128$ & 2.9 \\
\hline & Ceftriaxone & $\leq 0.50$ & $\leq 0.50$ & $\leq 0.50-128.0$ & 0.4 \\
\hline & Cefepime & $\leq 0.06$ & 0.12 & $\leq 0.06-0.50$ & 0 \\
\hline & Piperacillin & 16.0 & 512.0 & $\leq 1.0->512.0$ & 17.6 \\
\hline & Piperacillin/tazobactam & 4.0 & 32.0 & $\leq 1.0->512.0$ & 6.9 \\
\hline & Ticarcillin/clavulanate & 2.0 & 16.0 & $\leq 1.0->256.0$ & 2.7 \\
\hline & Imipenem & 0.50 & 1.0 & $\leq 0.06-4.0$ & 0 \\
\hline & Gentamicin & $\leq 4.0$ & $\leq 4.0$ & $\leq 4.0->16.0$ & 0.4 \\
\hline & Tobramycin & $\leq 4.0$ & $\leq 4.0$ & $\leq 4.0$ & 0 \\
\hline & Ciprofloxacin & $\leq 0.06$ & 0.12 & $\leq 0.06-0.50$ & 0 \\
\hline & TMP/SMX & 2.0 & 4.0 & $\leq 0.50->16.0$ & 10.9 \\
\hline \multirow{12}{*}{$\begin{array}{l}\text { Citrobacter freundii } \\
\quad(159)\end{array}$} & Cefotaxime & 0.25 & 16.0 & $\leq 0.06->64.0$ & 6.8 \\
\hline & Ceftazidime & $\leq 0.50$ & 32.0 & $\leq 0.50->128.0$ & 13.9 \\
\hline & Ceftriaxone & $\leq 0.50$ & 32.0 & $\leq 0.50->128.0$ & 7.8 \\
\hline & Cefepime & $\leq 0.06$ & 0.25 & $\leq 0.06-2.0$ & 0 \\
\hline & Piperacillin & 8.0 & 512.0 & $\leq 1.0->512.0$ & 18.4 \\
\hline & Piperacillin/tazobactam & 4.0 & 64.0 & $\leq 1.0-256.0$ & 5.1 \\
\hline & Ticarcillin/clavulanate & 2.0 & 128.0 & $\leq 1.0->256.0$ & 12.9 \\
\hline & Imipenem & 1.0 & 4.0 & $\leq 0.06-4.0$ & 0 \\
\hline & Gentamicin & $\leq 4.0$ & $\leq 4.0$ & $\leq 4.0->16.0$ & 2.6 \\
\hline & Tobramycin & $\leq 4.0$ & $\leq 4.0$ & $\leq 4.0->16.0$ & 1.7 \\
\hline & Ciprofloxacin & $\leq 0.06$ & $\leq 0.06$ & $\leq 0.06-8.0$ & 1.7 \\
\hline & TMP/SMX & 1.0 & $>16.0$ & $\leq 0.50->16.0$ & 21.6 \\
\hline \multirow{12}{*}{$\begin{array}{l}\text { Proteus vulgaris } \\
\text { (23) }\end{array}$} & Cefotaxime & 0.50 & 2.0 & $\leq 0.06-8.0$ & 0 \\
\hline & Ceftazidime & $\leq 0.50$ & $\leq 0.50$ & $\leq 0.50-2.0$ & 0 \\
\hline & Ceftriaxone & $\leq 0.50$ & 64.0 & $\leq 0.50-64.0$ & 12.7 \\
\hline & Cefepime & 0.50 & 0.50 & $\leq 0.06-0.50$ & 0 \\
\hline & Piperacillin & 2.0 & 4.0 & $\leq 1.0-128.0$ & 6.2 \\
\hline & Piperacillin/tazobactam & 2.0 & 2.0 & $\leq 1.0-4.0$ & 0 \\
\hline & Ticarcillin/clavulanate & 2.0 & $>256.0$ & $2.0->256.0$ & 6.4 \\
\hline & Imipenem & 2.0 & 2.0 & $0.50-4.0$ & 0 \\
\hline & Gentamicin & $\leq 4.0$ & $\leq 4.0$ & $\leq 4.0->16.0$ & 8.7 \\
\hline & Tobramycin & $\leq 4.0$ & $\leq 4.0$ & $\leq 4.0-8.0$ & 0 \\
\hline & Ciprofloxacin & $\leq 0.06$ & $\leq 0.06$ & $\leq 0.06-0.12$ & 0 \\
\hline & TMP/SMX & 2.0 & 16.0 & $\leq 0.50->16.0$ & 22.7 \\
\hline
\end{tabular}

${ }^{*}$ Minimum inhibitory concentration (MIC) for 50 and $90 \%$ of isolates tested. TMP/SMX Trimethoprim-sulfamethoxazole 
TABLE 1 (continued)

\begin{tabular}{|c|c|c|c|c|c|}
\hline \multirow{2}{*}{$\begin{array}{l}\text { Organism } \\
\text { (number) }\end{array}$} & \multirow[b]{2}{*}{ Antimicrobial agent } & \multicolumn{3}{|c|}{$\operatorname{MIC}(\mu \mathrm{g} / \mathrm{mL})^{*}$} & \multirow[b]{2}{*}{ Resistant (\%) } \\
\hline & & $50 \%$ & $90 \%$ & Range & \\
\hline \multirow{12}{*}{$\begin{array}{l}\text { Proteus mirabilis } \\
\text { (16) }\end{array}$} & Cefotaxime & 0.50 & 0.50 & $\leq 0.06->64.0$ & 3.3 \\
\hline & Ceftazidime & $\leq 0.50$ & $\leq 0.50$ & $\leq 0.50-64.0$ & 1.4 \\
\hline & Ceftriaxone & $\leq 0.50$ & $\leq 0.50$ & $\leq 0.50-2.0$ & 0 \\
\hline & Cefepime & 0.50 & 0.50 & $\leq 0.06-1.0$ & 0 \\
\hline & Piperacillin & 2.0 & 8.0 & $\leq 1.0->512.0$ & 18.8 \\
\hline & Piperacillin/tazobactam & 2.0 & 2.0 & $\leq 1.0-2.0$ & 0 \\
\hline & Ticarcillin/clavulanate & 2.0 & 2.0 & $2.0->256.0$ & 1.4 \\
\hline & Imipenem & 1.0 & 4.0 & $0.50-8.0$ & 6.2 \\
\hline & Gentamicin & $\leq 4.0$ & $\leq 4.0$ & $\leq 4.0->16.0$ & 8.7 \\
\hline & Tobramycin & $\leq 4.0$ & $\leq 4.0$ & $\leq 4.0-8.0$ & 0 \\
\hline & Ciprofloxacin & $\leq 0.06$ & $\leq 0.06$ & $\leq 0.06-0.12$ & 0 \\
\hline & TMP/SMX & 2.0 & 16.0 & $\leq 0.50->16.0$ & 22.7 \\
\hline \multirow{12}{*}{$\begin{array}{l}\text { Providencia species } \\
\text { (10) }\end{array}$} & Cefotaxime & 1.0 & 4.0 & $\leq 0.50-4.0$ & 0 \\
\hline & Ceftazidime & $\leq 0.50$ & 1.0 & $\leq 0.50-16.0$ & 0 \\
\hline & Ceftriaxone & $\leq 0.50$ & $\leq 0.50$ & $\leq 0.50-4.0$ & 0 \\
\hline & Cefepime & $\leq 0.06$ & 0.50 & $\leq 0.06-0.50$ & 0 \\
\hline & Piperacillin & 2.0 & 32.0 & $<1.0-512.0$ & 10.0 \\
\hline & Piperacillin/tazobactam & $\leq 1.0$ & 4.0 & $\leq 1.0-8.0$ & 0 \\
\hline & Ticarcillin/clavulanate & 2.0 & 16.0 & $\leq 1.0-16.0$ & 0 \\
\hline & Imipenem & 1.0 & 8.0 & $\leq 0.06-16.0$ & 10.0 \\
\hline & Gentamicin & 8.0 & $>16$ & $\leq 4.0->16.0$ & 40.0 \\
\hline & Tobramycin & $\leq 4.0$ & $\leq 4.0$ & $\leq 4.0$ & 0 \\
\hline & Ciprofloxacin & $\leq 0.06$ & $\leq 0.06$ & $\leq 0.06$ & 0 \\
\hline & TMP/SMX & 16.0 & $>16.0$ & $\leq 0.50->16.0$ & 40.0 \\
\hline \multirow{12}{*}{$\begin{array}{l}\text { Enterobacter } \\
\text { cloacae (550) }\end{array}$} & Cefotaxime & 1.0 & $>64.0$ & $\leq 0.06->64.0$ & 16.8 \\
\hline & Ceftazidime & 8.0 & $>128.0$ & $\leq 0.50->128.0$ & 28.9 \\
\hline & Ceftriaxone & $\leq 0.5$ & $>128.0$ & $\leq 0.5->128.0$ & 18.5 \\
\hline & Cefepime & 0.12 & 2.0 & $\leq 0.06-8.0$ & 0.2 \\
\hline & Piperacillin & 16.0 & 512.0 & $\leq 1.0->512.0$ & 32.4 \\
\hline & Piperacillin/tazobactam & 8.0 & 256.0 & $\leq 1.0->512.0$ & 16.9 \\
\hline & Ticarcillin/clavulanate & 32.0 & $>256.0$ & $\leq 1.0->256.0$ & 43.6 \\
\hline & Imipenem & 1.0 & 4.0 & $\leq 0.06 \rightarrow 64.0$ & 1.1 \\
\hline & Gentamicin & $\leq 4.0$ & 8.0 & $\leq 4.0->16.0$ & 7.6 \\
\hline & Tobramycin & $\leq 4.0$ & $\leq 4.0$ & $\leq 4.0->16.0$ & 2.8 \\
\hline & Ciprofloxacin & $\leq 0.06$ & 0.12 & $\leq 0.06-8.0$ & 0.9 \\
\hline & TMP/SMX & 1.0 & 2.0 & $\leq 0.50->16.0$ & 7.8 \\
\hline \multirow{12}{*}{$\begin{array}{c}\text { Enterobacter } \\
\text { species }^{\dagger} \\
(132)\end{array}$} & Cefotaxime & 0.25 & 32.0 & $\leq 0.06->64.0$ & 7.7 \\
\hline & Ceftazidime & $\leq 0.50$ & 128.0 & $\leq 0.50->128.0$ & 18.9 \\
\hline & Ceftriaxone & 0.50 & 64.0 & $\leq 0.50->128.0$ & 10.3 \\
\hline & Cefepime & $\leq 0.06$ & 0.50 & $\leq 0.06-32.0$ & 0.8 \\
\hline & Piperacillin & 8.0 & 128.0 & $<1.0->512.0$ & 11.4 \\
\hline & Piperacillin/tazobactam & 8.0 & 64.0 & $\leq 1.0-256.0$ & 7.6 \\
\hline & Ticarcillin/clavulanate & 4.0 & 256.0 & $\leq 1.0-256.0$ & 22.2 \\
\hline & Imipenem & 4.0 & 8.0 & $\leq 0.06-16.0$ & 2.3 \\
\hline & Gentamicin & $\leq 4.0$ & $\leq 4.0$ & $\leq 4.0->16.0$ & 3.4 \\
\hline & Tobramycin & $\leq 4.0$ & $\leq 4.0$ & $\leq 4.0->16.0$ & 3.4 \\
\hline & Ciprofloxacin & $\leq 0.06$ & 1.0 & $\leq 0.06-8.0$ & 5.1 \\
\hline & TMP/SMX & $\leq 0.50$ & 2.0 & $\leq 0.50->16.0$ & 8.5 \\
\hline
\end{tabular}

${ }^{*}$ Minimum inhibitory concentration (MIC) for 50 and $90 \%$ of isolates tested. ${ }^{\dagger}$ Includes 68 isolates of Enterobacter aerogenes, 27 isolates of Enterobacter agglomerans and 39 Enterobacter species. TMP/SMX Trimethoprim-sulfamethoxazole 
TABLE 1 (continued)

\begin{tabular}{|c|c|c|c|c|c|}
\hline \multirow{2}{*}{$\begin{array}{l}\text { Organism } \\
\text { (number) }\end{array}$} & \multirow[b]{2}{*}{ Antimicrobial agent } & \multicolumn{3}{|c|}{$\operatorname{MIC}(\mu \mathrm{g} / \mathrm{mL})^{*}$} & \multirow[b]{2}{*}{ Resistant (\%) } \\
\hline & & $50 \%$ & $90 \%$ & Range & \\
\hline \multirow{12}{*}{$\begin{array}{l}\text { Serratia marcescens } \\
\text { (189) }\end{array}$} & Cefotaxime & 0.25 & 2.0 & $\leq 0.06-64.0$ & 0.7 \\
\hline & Ceftazidime & $\leq 0.50$ & $\leq 0.50$ & $\leq 0.50-32.0$ & 0.5 \\
\hline & Ceftriaxone & $\leq 0.50$ & 1.0 & $\leq 0.50-32.0$ & 0 \\
\hline & Cefepime & $\leq 0.06$ & 0.25 & $\leq 0.06-16.0$ & 0 \\
\hline & Piperacillin & 8.0 & $>512.0$ & $\leq 1.0->512.0$ & 28.2 \\
\hline & Piperacillin/tazobactam & 2.0 & 16.0 & $\leq 1.0->512.0$ & 1.6 \\
\hline & Ticarcillin/clavulanate & 8.0 & 128.0 & $\leq 1.0->256.0$ & 14.0 \\
\hline & Imipenem & 2.0 & 4.0 & $\leq 0.06-8.0$ & 2.6 \\
\hline & Gentamicin & $\leq 4.0$ & 16.0 & $\leq 4.0->16.0$ & 26.2 \\
\hline & Tobramycin & $\leq 4.0$ & 16.0 & $\leq 4.0->16.0$ & 22.1 \\
\hline & Ciprofloxacin & $\leq 0.06$ & 0.50 & $\leq 0.06-8.0$ & 2.7 \\
\hline & TMP/SMX & 2.0 & 8.0 & $\leq 0.50->16.0$ & 31.3 \\
\hline \multirow{12}{*}{$\begin{array}{l}\text { Morganella morganii } \\
\quad(57)\end{array}$} & Cefotaxime & 0.12 & 16.0 & $\leq 0.06-16.0$ & 0 \\
\hline & Ceftazidime & $\leq 0.50$ & 32.0 & $\leq 0.50->128.0$ & 10.5 \\
\hline & Ceftriaxone & 0.50 & 2.0 & $\leq 0.50-32.0$ & 0 \\
\hline & Cefepime & $\leq 0.06$ & 0.12 & $\leq 0.06-0.25$ & 0 \\
\hline & Piperacillin & 4.0 & 256.0 & $\leq 1.0->512.0$ & 17.5 \\
\hline & Piperacillin/tazobactam & $\leq 1.0$ & 32.0 & $\leq 1.0-256.0$ & 3.5 \\
\hline & Ticarcillin/clavulanate & 4.0 & 32.0 & $\leq 1.0-128.0$ & 4.2 \\
\hline & Imipenem & 8.0 & 16.0 & $\leq 0.06-16.0$ & 28.0 \\
\hline & Gentamicin & $\leq 4.0$ & $\leq 4.0$ & $\leq 4.0-8.0$ & 0 \\
\hline & Tobramycin & $\leq 4.0$ & $\leq 4.0$ & $\leq 4.0-16.0$ & 2.1 \\
\hline & Ciprofloxacin & $\leq 0.06$ & 0.12 & $\leq 0.06-0.12$ & 0 \\
\hline & TMP/SMX & 2.0 & $>16.0$ & $\leq 0.50->16.0$ & 14.6 \\
\hline \multirow{12}{*}{$\begin{array}{l}\text { Pseudomonas } \\
\text { aeruginosa } \\
(228)\end{array}$} & Cefotaxime & 32.0 & $>64.0$ & $\leq 0.06->64.0$ & 43.2 \\
\hline & Ceftazidime & 4.0 & 32.0 & $\leq 0.50->128.0$ & 11.7 \\
\hline & Ceftriaxone & 32.0 & $>128.0$ & $\leq 0.50->128.0$ & 32.2 \\
\hline & Cefepime & 2.0 & 16.0 & $\leq 0.06-64.0$ & 28.6 \\
\hline & Piperacillin & 16.0 & 512.0 & $\leq 1.0->512.0$ & 12.6 \\
\hline & Piperacillin/tazobactam & 8.0 & 128.0 & $\leq 1.0->512.0$ & 10.9 \\
\hline & Ticarcillin/clavulanate & 32.0 & 128.0 & $\leq 1.0->256.0$ & 10.3 \\
\hline & Imipenem & 4.0 & 64.0 & $\leq 0.06-64.0$ & 23.3 \\
\hline & Gentamicin & $\leq 4.0$ & 16.0 & $\leq 4.0->16.0$ & 15.1 \\
\hline & Tobramycin & $\leq 4.0$ & $\leq 4.0$ & $\leq 4.0->16.0$ & 2.7 \\
\hline & Ciprofloxacin & 0.25 & 2.0 & $\leq 0.06-32.0$ & 4.8 \\
\hline & TMP/SMX & $>16.0$ & $>16.0$ & $>16.0$ & 100 \\
\hline
\end{tabular}

${ }^{*}$ Minimum inhibitory concentration (MIC) for 50 and $90 \%$ of isolates tested. TMP/SMX Trimethoprim-sulfamethoxazole

Isolates of $E$ coli, K pneumoniae and $P$ mirabilis were more susceptible to piperacillin/tazobactam (MIC90 $32.0 \mathrm{~g} / \mathrm{mL}$ ) than those with type 1 cephalosporinases. Of the isolates of Enterobacteriaceae and $P$ aeruginosa resistant to piperacillin alone, $290(16.6 \%)$ and 34 (14.8\%), respectively, became sensitive when piperacillin was combined with tazobactam.

For all isolates tested, the most active agents were the aminoglycosides, imipenem and ciprofloxacin. Ciprofloxacin resistance was less than $2.0 \%$ in all isolates of Enterobacteriaceae with the notable exception of Enterobacter species, in which $5.1 \%$ of isolates had MICs to ciprofloxacin of greater than $4.0 \mathrm{~g} / \mathrm{mL}$.

Imipenem exhibited excellent in vitro activity. Resistance was detected only in $P$ aeruginosa (23.3\%) and Enterobacter species $(2.3 \%)$. The highest incidence of aminoglycoside resistance was found in isolates of $S$ marcescens, in which $26.2 \%$ and $22.1 \%$ of isolates were gentamicin- and tobramycin-resistant, respectively. In all other species tested, including $P$ aeruginosa, aminoglycoside resistance was less than $10 \%$. Tobramycin was much more effective against isolates of $P$ aeruginosa than gentamicin, because only $2.7 \%$ were resistant to this aminoglycoside whereas $15.1 \%$ of isolates were resistant to gentamicin.

\section{DISCUSSION}

The rapid initiation of effective antimicrobial therapy for the 
management of infections caused by Gram-negative bacilli can significantly improve outcome (24). Treatment is often empirical and based primarily on knowledge of the antimicrobial susceptibility of the most common bacteria encountered. It is therefore crucial to conduct broadly based surveillance studies to generate susceptibility data that can help predict susceptibility of a species to an antimicrobial or group of antimicrobials. However, it is also necessary to know or highly suspect what species are responsible for the disease syndrome in a given patient population.

In light of the increasing importance of Enterobacter species as important nosocomial pathogens, changes in the beta-lactam susceptibility profile of these isolates are of great concern. Sanders (12) found that, although all isolates of $E$ cloacae will produce a BGI beta-lactamase on induction, $28.9 \%$ of these isolates have become constitutive producers.

Cefepime, a fourth-generation cephalosporin, is structurally similar to cefotaxime, ceftriaxone and ceftizoxime. However, a quaternary nitrogen-containing substitution on the positively charged third carbon and the negative charge on position four of the cephalosporin nucleus gives cefepime zwitterionic properties that allow cefepime to penetrate very rapidly through the outer membrane of Gram-negative bacteria. As well, cefepime, like other fourth-generation cephalosporins, is stable against BGI beta-lactamases (13).

For all isolates, the most active cephalosporin tested was cefepime. Among Enterobacteriaceae, more than $98 \%$ of all isolates were susceptible to this agent. The activity of cefepime equalled or exceeded that of third-generation cephalosporins. Isolates demonstrating resistance to third-generation cephalosporins remained susceptible to cefepime. Major differences in activity between cefepime and the thirdgeneration cephalosporins were found in isolates with $\mathrm{BGI}$ beta-lactamases, ie, E cloacae, Enterobacter aerogenes, $C$ freundii, $M$ morganii and $S$ marcescens. Against $P$ aeruginosa, cefepime and ceftazidime were more active than cefotaxime, but these differences are likely the result of different permeabilities for these agents (25) as opposed to betalactamase stability.

For all isolates tested, the activity of piperacillin was highly potentiated when combined with the beta-lactamase inhibitor tazobactam. The beta-lactam/beta-lactamase inhibitor combination of piperacillin/tazobactam was more effective than ticarcillin/clavulanate against isolates possessing $\mathrm{BGI}$ beta-lactamases, isolates found in this study and others to be increasingly resistant to traditional third-generation therapeutic agents.

Antimicrobial resistance among isolates of $K$ pneumoniae and $E$ coli occurred infrequently. Resistance to thirdgeneration cephalosporins was rare, which contrasts sharply with reports from Europe and specific areas of the United States, where the prevalence of resistance is high with the occurrence of nosocomial outbreaks $(7,8)$.

\section{CONCLUSIONS}

This study demonstrated that third-generation cephalosporin resistance is a clinically relevant problem in isolates known to possess BGI beta-lactamases. Although crossresistance to other third-generation cephalosporins was seen, cefepime and nonbeta-lactam agents retained their activity. Plasmid-mediated extended spectrum beta-lactamase was not a significant finding. Traditional nonbeta-lactam agents were found to have retained their activity against nosocomial Gram-negative rods. As well, aminoglycosides are still highly active against the majority of isolates tested, with the notable exception of $S$ marcescens.

\section{REFERENCES}

1. Bush K. Characterization of $\beta$-lactamases. Antimicrob Agents Chemother 1989;33:259-63.

2. Knothe HP, Shah V, Kremery M, et al. Transferable resistance to cefotaxime, cefoxitin, cefamandole, and cefuroxime in clinical isolates of Klebsiella pneumoniae and Serratia marcescens. Infection 1983;11:315-7.

3. Sanders CC. Chromosomal cephalosporinases responsible for multiple resistance to newer $\beta$-lactam antibiotics. Ann Rev Microbiol 1987;41:573-93.

4. Jacoby GA, Medeiros AA. More extended spectrum $\beta$-lactamases. Antimicrob Agents Chemother 1991;35:1697-704.

5. Papanicolaou GA, Medeiros AA, Jacoby GA. Novel plasmid-mediated $\beta$-lactamase (MIR-1) conferring resistance to oxyimino- and $\alpha$-methoxy $\beta$-lactams in clinical isolates of Klebsiella pneumoniae. Antimicrob Agents Chemother 1990;34:2200-9.

6. Phillipon A, Labia R, Jacoby GA. Extended spectrum $\beta$-lactamases. Antimicrob Agents Chemother 1989;33:1131-6.

7. Quinn JP, Miyashiro D, Sahm D, et al. Novel plasmid-mediated $\beta$-lactamase (TEM-10) conferring resistance to ceftazidime and aztreonam in clinical isolates of Klebsiella pneumoniae. Antimicrob Agents Chemother 1989;33:1451-6.

8. Rice LB, Willey SH, Papanicolaou GA, et al. Outbreak of ceftazidime resistance caused by extended spectrum $\beta$-lactamases at a Massachusetts chronic care facility. Antimicrob Agents Chemother 1990;34:2193-9.

9. Aronoff SC, Shlaes DM. Factors that influence the evolution of $\beta$-lactam resistance in $\beta$-lactamase-inducible strains of Enterobacter cloacae and Pseudomonas aeruginosa. J Infect Dis 1987;155:936-41.

10. Jones RN. The current and future impact of antimicrobial resistance among nosocomial bacterial pathogens. Diagn Microbiol Infect Dis 1992;15:3S-10S.

11. Livermore DM. Mechanisms of resistance to $\beta$-lactam antibiotics. Scand J Infect Dis 1991;78(Suppl):7-16.

12. Sanders CC. $\beta$-Lactamases of Gram negative bacteria: new challenges for new drugs. Clin Infect Dis 1992;14:1089-99.

13. Sanders CC. Cefepime: the next generation. Clin Infect Dis 1993;17:369-79.

14. Thornsberry C, Brown SD, Yee YC, et al. In vitro activity of cefepime and other antimicrobials: survey of European isolates. J Antimicrob Chemother 1993;32(Suppl B):31-53.

15. Duval J, Soussy CJ, Acar JF, et al. In vitro antibacterial activity of cefepime: a multicentre study. J Antimicrob Chemother 1993;32(Suppl B):55-61.

16. Toye BW, Scriver SR, Low DE, et al. Canadian survey of antimicrobial resistance in Klebsiella spp and Enterobacter spp. J Antimicrob Chemother 1993;32(Suppl B):81-6.

17. Aronoff SC, Jacobs MR, Johenning S, et al. Comparative activities of the $\beta$-lactamase inhibitors YTR 830 , sodium clavulanate and sulbactam combined with amoxicillin or ampicillin. Antimicrob Agents Chemother 1984;26:580.

18. Appelbaum PC, Jacobs MR, Spangler SR, et al. Comparative activity of the $\beta$-lactamase inhibitor YTR 830 , clavulanate and sulbactam combined with $\beta$-lactams against $\beta$-lactamase 
producing anaerobes. Antimicrob Agents Chemother 1986;30:798.

19. Gutmann L, Kitzis MD, Yamabi S, et al. Comparative evaluation of a new b-lactamase inhibitor YTR 830, combined with different b-lactam antibiotics against bacteria harbouring known b-lactamases. Antimicrob Agents Chemother 1986;29:955.

20. Jacobs MR, Aronoff SC, Jownning S, et al. Comparative activities of the b-lactamase inhibitors YTR 830, clavulanate and sulbactam combined with extended spectrum penicillins against ticarcillin-resistant Enterobacteriaceae and pseudomonads. J Antimicrob Chemother 1986;18:177.

21. Moosdeen F, Williams JD, Yamabe S. Antibacterial characteristics of YTR 830, a sulfone b-lactamase inhibitor, compared with those of clavulanic acid and sulbactam. Antimicrob Agents Chemother 1988;32:925.
22. Farmer JJ, Kelly MT. Enterobacteriaceae. In: Balows A, Hausler WJ, Herrmann KL, et al, eds. Manual of Clinical Microbiology, 5th edn. Washington: American Society for Microbiology, 1991:360-83.

23. National Committee for Clinical Laboratory Standards. Methods for Dilution Antimicrobial Susceptibility Tests for Bacteria that Grow Aerobically. Approved Standard M7-A3. Villanova: National Committee for Clinical Laboratory Standards, 1993.

24. Gransden WR, Elykyn SJ, Phillips I, et al. Bacteremia due to Escherichia coli: a study of 861 episodes. Rev Infect Dis 1990;12:1008-18.

25. Pucci MF, Boice-Sowek J, Kessler RE, et al. Comparison of cefepime, cefpirome and cefaclidine binding affinities for penicillin binding proteins in Escherichia coli K-12 and Pseudomonas aeruginosa SC8329. Antimicrob Agents Chemother 1991;35:2312-7. 


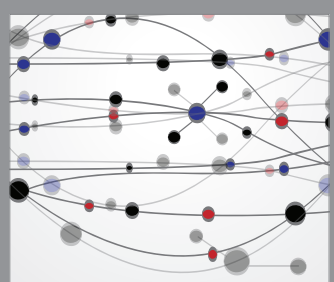

The Scientific World Journal
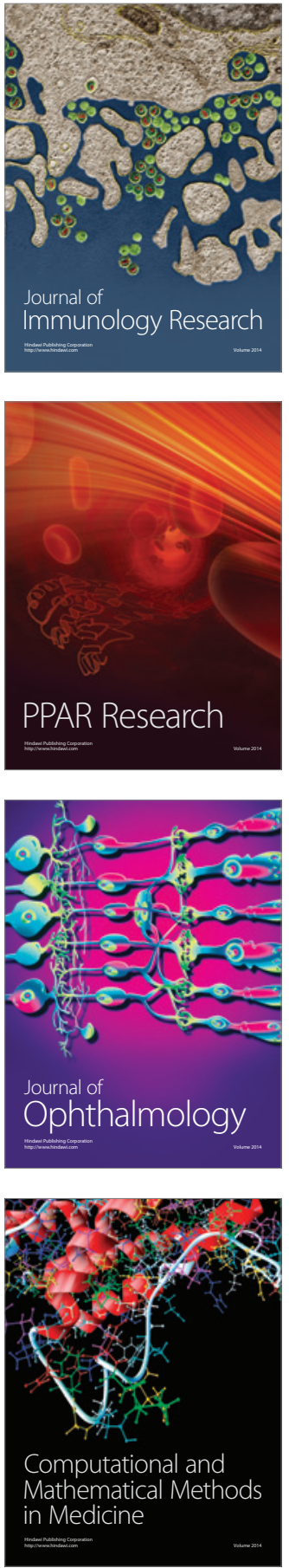

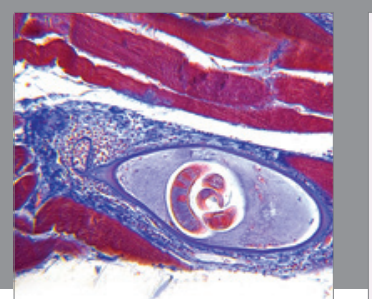

Gastroenterology Research and Practice

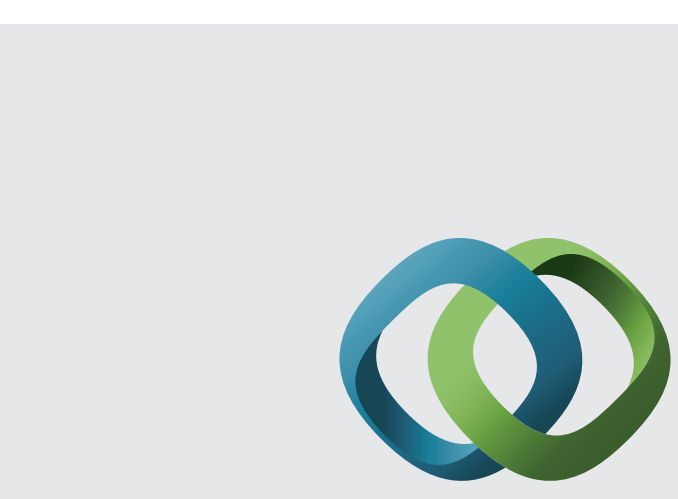

\section{Hindawi}

Submit your manuscripts at

http://www.hindawi.com
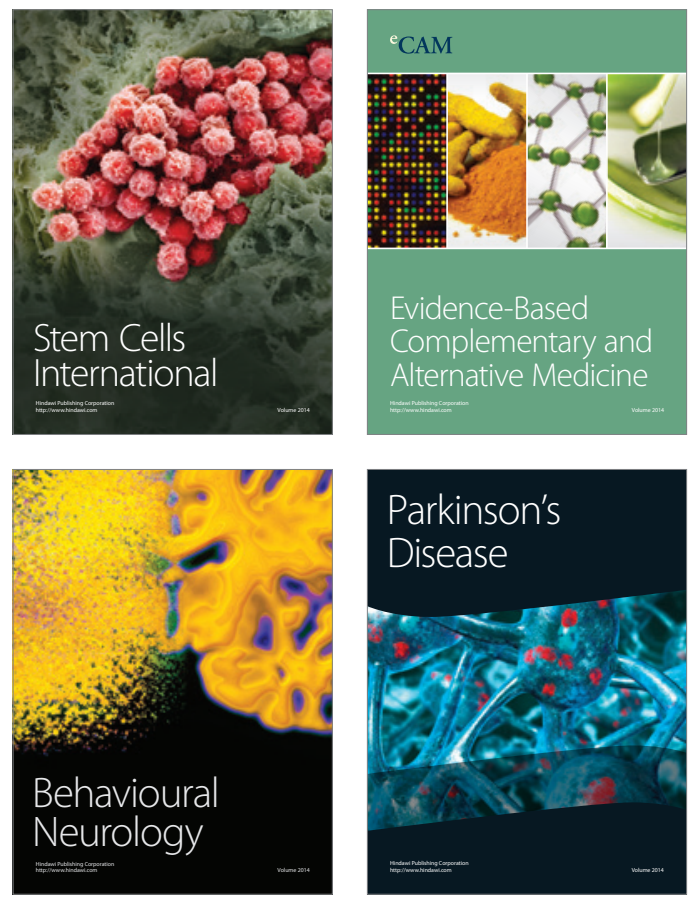
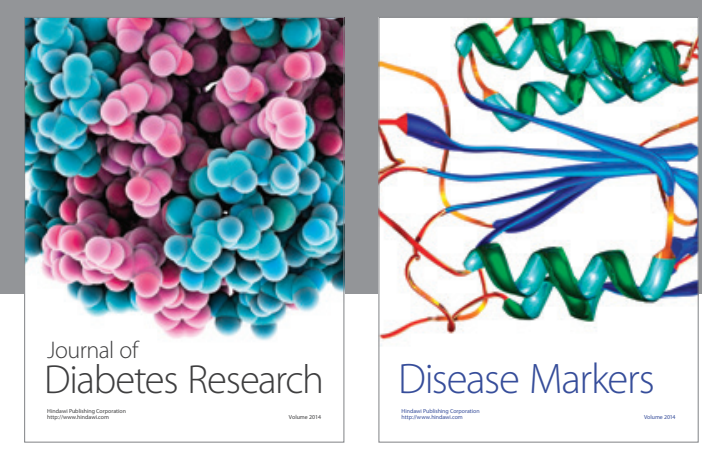

Disease Markers
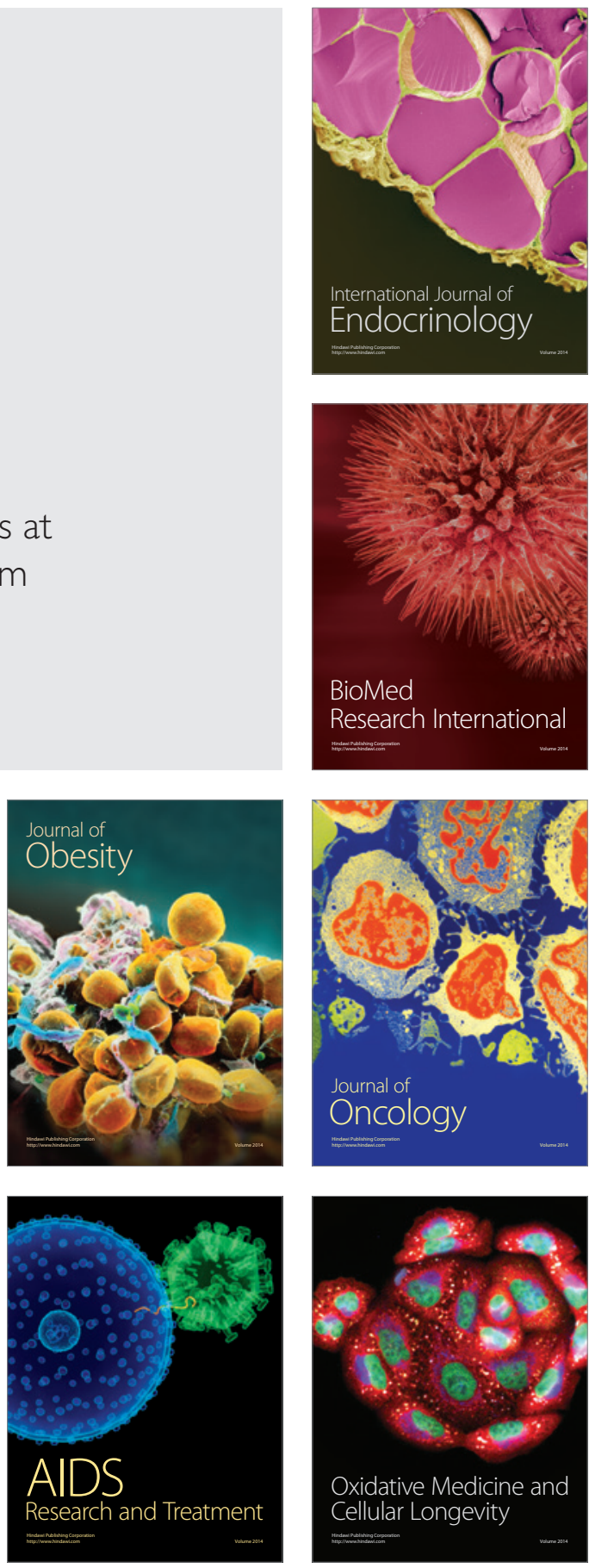